\title{
$(\mathrm{GeTe})_{n} \mathrm{Bi}_{2} \mathrm{Te}_{3}$ 的结构与热电性能研究
}

\author{
杨 香，苏贤礼，跒永高，唐新峰 \\ (武汉理工大学 材料复合新技术国家重点实验室, 武汉 430070)
}

摘 要: 在 $\mathrm{GeTe}-\mathrm{Bi}_{2} \mathrm{Te}_{3}$ 赝二元系统中, $(\mathrm{GeTe})_{n}\left(\mathrm{Bi}_{2} \mathrm{Te}_{3}\right)_{m}$ 化合物往往具有较低的晶格热导率, 但其中很多组分的热电 性能尚未得到系统研究。本研究通过熔融、淬火、退火结合放电等离子烧结工艺制备了一系列 $(\mathrm{GeTe})_{n} \mathrm{Bi}_{2} \mathrm{Te}_{3}(n=10$, $11,12,13,14)$ 单相多晶样品, 并对其相组成和热电性能进行表征和研究。掺杂 $\mathrm{Bi}_{2} \mathrm{Te}_{3}$ 可以显著增强点缺陷声子散射, 大幅度降低材料的晶格热导率, 在 $723 \mathrm{~K}$ 时, $(\mathrm{GeTe})_{13} \mathrm{Bi}_{2} \mathrm{Te}_{3}$ 样品的总热导率低至 $1.63 \mathrm{~W} \cdot \mathrm{m}^{-1} \cdot \mathrm{K}^{-1}$ 。此外, 掺杂 $\mathrm{Bi}_{2} \mathrm{Te}_{3}$ 和调控 $\mathrm{GeTe}$ 的相对含量, 提高了材料的载流子有效质量, 即使在较高的载流子浓度下, 样品依然保持较高的塞贝 克系数和功率因子, 在 $723 \mathrm{~K},(\mathrm{GeTe})_{13} \mathrm{Bi}_{2} \mathrm{Te}_{3}$ 样品获得最大的功率因子为 $2.88 \times 10^{-3} \mathrm{~W} \cdot \mathrm{m}^{-1} \cdot \mathrm{K}^{-2}$, 最终 $\left(\mathrm{GeTe}_{13} \mathrm{Bi}_{2} \mathrm{Te}_{3}\right.$ 样品在 $723 \mathrm{~K}$ 获得的最大 $Z T$ 值达到 1.27 , 较未掺杂的 GeTe 样品提高了 $16 \%$ 。

关 键 词: $\mathrm{GeTe} ; \mathrm{Bi}_{2} \mathrm{Te}_{3}$ 掺杂; 结构; 热电性能

中图分类号: TB34/O482.6 文献标识码: A

\section{Structures and Thermoelectric Properties of $(\mathrm{GeTe})_{n} \mathrm{Bi}_{2} \mathrm{Te}_{3}$}

\author{
YANG Xiao, SU Xianli, YAN Yonggao, TANG Xinfeng
}

(State Key Laboratory of Advanced Technology for Materials Synthesis and Processing, Wuhan University of Technology, Wuhan 430070, China)

Abstract: In general, $(\mathrm{GeTe})_{n}\left(\mathrm{Bi}_{2} \mathrm{Te}_{3}\right)_{m}$ compounds in $\mathrm{GeTe}-\mathrm{Bi}_{2} \mathrm{Te}_{3}$ pseudo-binary system possess a relatively low thermal conductivity, however, the thermoelectric properties of these compounds have not been evaluated systematically. In this study, a series of single-phase $\left(\mathrm{GeTe}_{n} \mathrm{Bi}_{2} \mathrm{Te}_{3}(n=10,11,12,13,14)\right.$ compounds were prepared by a melting-quenching-annealing process combined with spark plasma sintering. The phase compositions and thermoelectrical properties of these samples were characterized. It is found that doping with $\mathrm{Bi}_{2} \mathrm{Te}_{3}$ intensifies the phonon scattering and significantly reduces the lattice thermal conductivities of these samples, producing a low total thermal conductivity of $1.63 \mathrm{~W} \cdot \mathrm{m}^{-1} \cdot \mathrm{K}^{-1}$ at $723 \mathrm{~K}$ for $(\mathrm{GeTe})_{13} \mathrm{Bi}_{2} \mathrm{Te}_{3}$ compound. Moreover, the effective mass of these compounds is enhanced through adjustment of the relative amount of $\mathrm{Bi}_{2} \mathrm{Te}_{3}$ and GeTe. Therefore, the Seebeck coefficient and power factor of these samples remain superior even at high carrier concentration. At $723 \mathrm{~K}$, the maximum power factor of $(\mathrm{GeTe})_{13} \mathrm{Bi}_{2} \mathrm{Te}_{3}$ compound is $2.88 \times 10^{-3} \mathrm{~W} \cdot \mathrm{m}^{-1} \cdot \mathrm{K}^{-2}$ and the maximum $Z T$ of $(\mathrm{GeTe})_{13} \mathrm{Bi}_{2} \mathrm{Te}_{3}$ is 1.27 , which is $16 \%$ higher than that of pristine GeTe.

Key words: $\mathrm{GeTe} ; \mathrm{Bi}_{2} \mathrm{Te}_{3}$ doping; structure; thermoelectric property

随着社会经济的高速发展，人类对能源的消耗与日俱增。化石能源储量有限，日渐枯竭，由燃烧化

收稿日期：2020-05-12；收到修改稿日期：2020-06-13; 网络出版日期：2020-07-13

基金项目: 国家重点研发计划(2019YFA0704900) National Key R\&D Program of China(2019YFA0704900)

作者简介: 杨 香(1995-), 男, 硕士研究生. E-mail: YX Sean@whut.edu.cn

YANG Xiao(1995-), male, Master candidate. E-mail: YX_Sean@whut.edu.cn

通信作者：唐新峰，教授.E-mail: tangxf@whut.edu.cn

TANG Xinfeng, professor. E-mail: tangxf@whut.edu.cn 
石能源导致的环境问题也日益严重。此外, 燃烧化 石能源所释放的大部分能量以低品位废热的形式散 失到大气中, 对这些废热进行有效地回收利用、提 高能源利用率, 具有重大的经济价值。热电材料作 为一种能够实现热能与电能相互转换的功能材料引 起了研究者的广泛关注, 在温差发电和热电制冷领 域表现出广泛的应用前景。热电器件的转换效率一 般由与热电材料的性能直接相关的无量纲热电优值 $Z T=\alpha^{2} \sigma T / \kappa$ 来衡量, 其中 $\alpha$ 为材料的塞贝克系数, $\sigma$ 为电导率, $\kappa$ 为热导率, 因此性能优异的热电材料需 要同时具有高的电导率、大的塞贝克系数和尽可能 低的热导率 ${ }^{[1]}$ 。

$\mathrm{GeTe}$ 基化合物作为一类性能优异的中温区热电 材料得到了广泛研究 ${ }^{[2-4]}$ 。GeTe 基化合物在室温下 为菱方相 $(\mathrm{R} 3 \mathrm{~m})$ 结构, 随着温度的升高, 在 $700 \mathrm{~K}$ 左 右发生相变, 转变为高温立方岩盐相结构 $(\mathrm{Fm}-3 \mathrm{~m})^{[5]}$ 。 由于其易产生大量本征 $\mathrm{Ge}$ 空位，具有较高的载流 子浓度 $\left(10^{21} \mathrm{~cm}^{-3}\right)^{[6]}$, 本征 $\mathrm{GeTe}$ 样品的热导率和电 导率均较高, 而塞贝克系数较小, 导致本征 $\mathrm{GeTe}$ 热 电性能不佳 ${ }^{[7]}$ 。目前, 可以通过化学计量比调控来抑 制 Ge 空位的形成，降低材料的载流子浓度。如 2019 年, Dong 等 ${ }^{[8]}$ 通过调控 $\mathrm{Ge}$ 和 $\mathrm{Te}$ 的化学计量比例, 对 基体中加入过量的 $\mathrm{Ge}$, 有效抑制产生 $\mathrm{Ge}$ 空位, 显 著降低空穴载流子浓度, 大幅度提高了材料的热电 性能。此外, 还可以掺杂异价施主元素如 $\mathrm{Bi} 、 \mathrm{Sb}$ 等 或共掺杂来降低其载流子浓度 ${ }^{[9-11]}$ 。总之, GeTe 材 料中载流子浓度与材料中空位浓度和掺杂元素的量 等因素密切相关。

$(\mathrm{GeTe})_{2} \mathrm{Sb}_{2} \mathrm{Te}_{3}$ 作为一种相变信息存储材料已被 研究多年, 在晶态下具有较高的电导率和较低的热 导率 ${ }^{[12]}$, 是一种潜在的高性能热电材料。一系列如 $(\mathrm{GeTe})_{n}\left(\mathrm{M}_{2} \mathrm{Te}_{3}\right)_{m}(\mathrm{M}=\mathrm{Sb}$ 和 $\mathrm{Bi})$ 的噟二元化合物的热 电性能引起了研究者的关注, 这些材料具有与 $\mathrm{GeTe}$ 化合物相似的相转变过程, 且一般热导率较低。其中, $\mathrm{M}=\mathrm{Sb}$ 样品的热电性能已经进行了充分研究 ${ }^{[13-17]}$, 而在 $\mathrm{M}=\mathrm{Bi}$ 的方面, Shelimova 等 ${ }^{[18]}$ 对 $(\mathrm{GeTe})_{n} \mathrm{Bi}_{2} \mathrm{Te}_{3}$ $(n=2 \sim 9)$ 系列样品的热电性能进行了初步研究, 但主 要偏重于研究系列化合物的结构演变规律。Li 等 ${ }^{[19]}$ 对 $(\mathrm{GeTe})_{0.97}\left(\mathrm{Bi}_{2} \mathrm{Te}_{3}\right)_{0.03}$ 化合物的研究表明, 与只掺 杂施主元素 $\mathrm{Bi}$ 的样品相比, 以 $\mathrm{Bi}_{2} \mathrm{Te}_{3}$ 掺杂的噟二元 化合物，除了 $\mathrm{Bi}$ 置换进入 $\mathrm{Ge}$ 位外，同时还会产生 阳离子空位。由于这类材料的晶格中的 $\mathrm{Ge}$ 位被 $\mathrm{Ge}$ 、 $\mathrm{Bi}$ (或 $\mathrm{Sb}$ )和产生的阳离子空位按一定比例占据, 且 分布具有随机性, 引起的剧烈晶格畸变造成材料热 导率大幅下降。 $\mathrm{Wu}$ 等 ${ }^{[20]}$ 的研究指出, 掺杂 $\mathrm{Bi}_{2} \mathrm{Te}_{3}$ 除了能作为施主调节材料的载流子浓度之外, 还能
对相结构和能带结构起到优化作用，从而提高材料 的 $Z T$ 值。但是迄今为止, $(\mathrm{GeTe})_{n} \mathrm{Bi}_{2} \mathrm{Te}_{3}$ 在 $n>9$ 时的 组分还未得到系统研究。

本研究采用熔融、淬火、退火结合放电等离子 活化烧结(SPS)工艺合成了 $(\mathrm{GeTe})_{n} \mathrm{Bi}_{2} \mathrm{Te}_{3}(n=10 \sim 14)$ 系列样品, 系统研究了 $\mathrm{Bi}_{2} \mathrm{Te}_{3}$ 相对含量对材料的物 相组成和热电性能的影响规律。

\section{1 实验方法}

\section{$1.1(\mathrm{GeTe})_{n} \mathrm{Bi}_{2} \mathrm{Te}_{3}$ 块体材料的制备}

本研究采用熔融、淬火、退火结合放电等离子 活化烧结(SPS)工艺, 制备名义组成为 $(\mathrm{GeTe})_{n} \mathrm{Bi}_{2} \mathrm{Te}_{3}$ $(n=10,11,12,13,14)$ 的一系列多晶块体材料。将按 化学计量比称量的高纯度 $\mathrm{Ge}(6 \mathrm{~N}$ 颗粒) $、 \mathrm{Bi}(5 \mathrm{~N}$ 块体) 和 $\mathrm{Te}(5 \mathrm{~N}$ 块体)真空密封放入石英玻璃管中，在 $1173 \mathrm{~K}$ 下熔融 $24 \mathrm{~h}$ 后, 在过饱和盐水溶液中快速淬 火, 然后将淬火得到的样品在 $623 \mathrm{~K}$ 下退火 $100 \mathrm{~h}$ 。 将所得锭体破碎, 研磨成精细粉末, 再通过 SPS 烧结 $(773 \mathrm{~K}, 45 \mathrm{MPa})$ 得到致密的块体样品。将所得样品 切割成规定尺寸和形状，用于结构表征和性能测试。

\section{2 物相组成分析}

采用粉末 $\mathrm{X}$ 射线衍射 (XRD, PANalyticalEmpyrean, $\mathrm{Cu} \mathrm{K} \alpha$ ) 和附加能谱仪模块(EDS, INCA X-Act) 的电子探针微区分析仪(EPMA，JXA-8230) 测试和表征材料的物相组成。

\section{3 测试热电性能}

在 $300 \sim 773 \mathrm{~K}$ 范围内, 样品的塞贝克系数和电 导率由热电性能测试设备(Ulvac Riko, ZEM-3)测量; 样品的热导率通过公式 $\kappa=C_{\mathrm{p}} D \rho$ 计算, 其中, 比热容 $C_{\mathrm{p}}$ 由 Dulong-Petit 定律计算得到, 通过激光导热仪 (Netzsch, LFA 457)测得热扩散系数 $D$, 采用阿基米 德排水法测得样品的密度 $\rho$ 。样品的室温霍尔系数 $R_{\mathrm{H}}$ 通过物理性能测试系统 (Quantum Design, PPMS-9)测得, 载流子浓度 $n_{\mathrm{H}}$ 和霍尔迁移率 $\mu_{\mathrm{H}}$ 由公 式 $n_{\mathrm{H}}=-1 /\left(e R_{\mathrm{H}}\right)$ 和 $\mu_{\mathrm{H}}=\left|R_{\mathrm{H}}\right| \sigma$ 计算得到。

\section{2 结果与讨论}

\section{1 相组成表征}

为了获得样品的物相、组成信息, 本研究对所制 备的 $(\mathrm{GeTe})_{n} \mathrm{Bi}_{2} \mathrm{Te}_{3}$ 系列样品进行了 XRD 和 $\mathrm{EDS}$ 测试。

图 1 为 $(\mathrm{GeTe})_{n} \mathrm{Bi}_{2} \mathrm{Te}_{3}(n=10 \sim 14)$ 系列样品的 XRD 图谱。可以看出所有样品均为菱方相结构, 在 $2 \theta=$ $40^{\circ} \sim 45^{\circ}$ 角度范围内可以观测到菱方相结构中 $(024)$ 和(220)两个晶面的典型特征峰, 与 $\mathrm{GeTe}-\mathrm{Bi}_{2} \mathrm{Te}_{3}$ 噟 


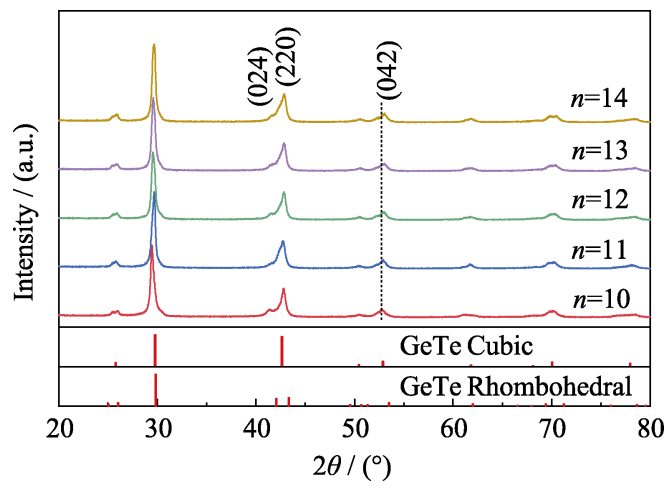

图 $1(\mathrm{GeTe})_{n} \mathrm{Bi}_{2} \mathrm{Te}_{3}(n=10 \sim 14)$ 化合物的粉末 $\mathrm{XRD}$ 图谱

Fig. 1 Powder XRD patterns of $\left(\mathrm{GeTe}_{n} \mathrm{Bi}_{2} \mathrm{Te}_{3}(n=10 \sim 14)\right.$ compounds

二元系中其他相邻组分相似 ${ }^{[18-19]}$, 说明在很宽的组 成范围内，在 $\mathrm{GeTe}$ 基材料中掺入的 $\mathrm{Bi}_{2} \mathrm{Te}_{3}$ 并未改变 材料的菱方相结构特征。另外, 随着 $\mathrm{GeTe}$ 含量的增 加, XRD 图谱的(042)晶面的峰位向大角度偏移, 说 明晶面间距减小, 这主要是因为 $\mathrm{Bi}$ 原子置换进入晶 格中 $\mathrm{Ge}$ 位, $\mathrm{Bi}$ 的原子半径大于 $\mathrm{Ge}$ 的原子半径, 与 形成的阳离子空位共同作用，导致晶体结构发生畸 变。这些结果表明 $\mathrm{Bi}$ 已经掺杂进入 $\mathrm{GeTe}$ 晶格。

由于所有样品的背散射电子图像和元素面扫描 结果基本一致, 在这里选取 $\mathrm{GeTe}$ 含量最高的 $(\mathrm{GeTe})_{14} \mathrm{Bi}_{2} \mathrm{Te}_{3}$ 和最低的 $(\mathrm{GeTe})_{10} \mathrm{Bi}_{2} \mathrm{Te}_{3}$ 两个样品为 代表，给出了它们的背散射电子和元素面分布图像, 分别如图 2(a d) 和图 2(e h) 所示。在样品的背散射 电子图像中，除了观测到少量因孔洞导致的明暗祄 度外，未观测到其他明显的明暗衬度，元素面分布 结果表明 $\mathrm{Ge} 、 \mathrm{Te} 、 \mathrm{Bi}$ 三种元素分布均匀, 未出现偏 析，与 XRD 的测试结果一致。这些测试结果表明, 采用熔融一退火结合 SPS 所制备的系列样品为元素 分布均匀的单相块体样品。
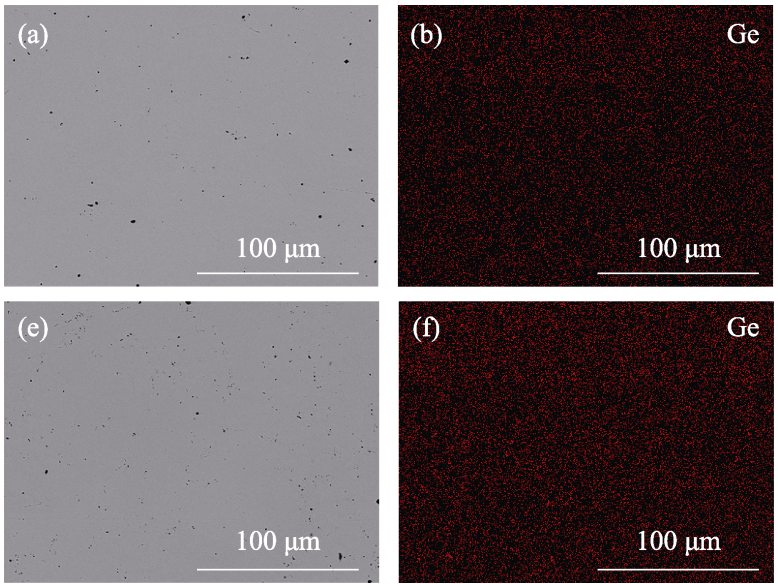

\section{2 电输运性能及分析}

图 3(a)为 $(\mathrm{GeTe})_{n} \mathrm{Bi}_{2} \mathrm{Te}_{3}$ 系列样品的电导率随温 度的变化曲线, 大致可以分为以 525 和 $625 \mathrm{~K}$ 两个 温度点分隔的三个温区，主要与材料的本征激发和 相变有关。当温度高于 $525 \mathrm{~K}$, 具有菱方相结构的样 品发生本征激发, 样品的电导率开始随温度升高而 增大。

在 $625 \mathrm{~K}$ 左右, 样品开始由菱方相结构转变为 立方相，电导率的上升趋势减缓，在 $650 \mathrm{~K}$ 左右达 到极大值。随着温度的进一步升高, 材料的电导率 下降，表现为金属传导特性。材料的塞贝克系数随 温度的变化如图 3(b)所示，在整个测试温度区间内， 塞贝克系数为正值, 系列样品均表现为 $\mathrm{p}$ 型传导, 并且变化趋势与电导率相反, 也可以按照上述的三 个温区来进行描述。在 $525 \mathrm{~K}$ 以下，塞贝克系数随 温度的升高而增大, 在 525 625 $\mathrm{K}$ 温度范围内塞贝 克系数出现平台区，样品的塞贝克系数随温度变化 较小, 这主要由于本征激发遏制了塞贝克系数的上 升, 在 $625 \mathrm{~K}$ 之后, 样品的塞贝克系数继续随温度 升高而增大, 在 $773 \mathrm{~K}$ 左右, $n=11$ 的样品获得最大 塞贝克系数 $\left(208 \mu \mathrm{V} \cdot \mathrm{K}^{-1}\right)$ 。此外, $n=11$ 的样品在 $725 \mathrm{~K}$ 后也出现了平台区，这与材料在立方相结构下的本 征激发有关。

为了更进一步了解样品的电输运机理, 通过样 品在室温下测试得到的霍尔系数和电导率计算得到 了样品的载流子浓度和迁移率。在假设声学声子散 射占主导的条件下，根据单抛物能带结构 SPB (Single Parabolic Band)模型, 经过简化, 材料的塞贝克系数 可以表示为:

$$
S=\frac{8 \pi^{2} k_{\mathrm{B}}^{2}}{3 e h^{2}} m^{*} T\left(\frac{\pi}{3 n}\right)^{2 / 3}
$$
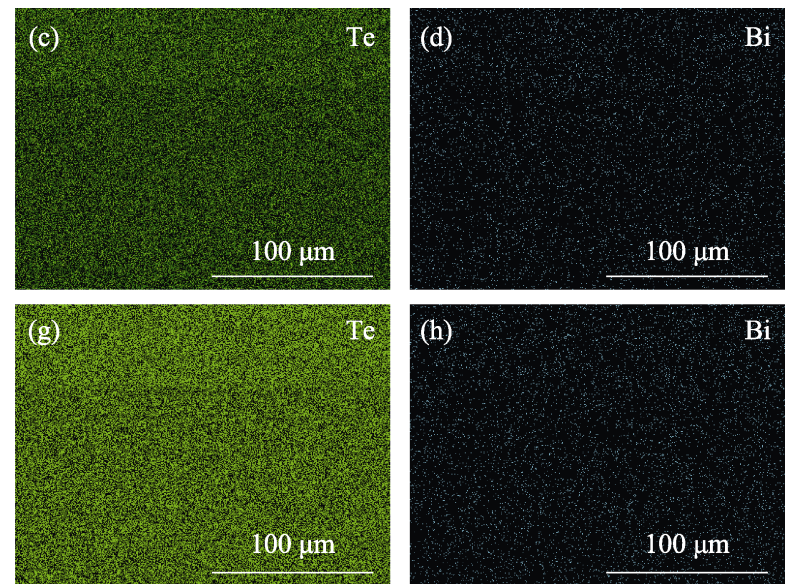

图 $2(\mathrm{GeTe})_{n} \mathrm{Bi}_{2} \mathrm{Te}_{3}$ 样品 $((\mathrm{a} \sim \mathrm{d}) n=10,(\mathrm{e} \sim \mathrm{h}) n=14)$ 抛光面的 $(\mathrm{a}, \mathrm{e})$ 背散射电子图像和 $(\mathrm{b}, \mathrm{f}) \mathrm{Ge},(\mathrm{c}, \mathrm{g}) \mathrm{Te},(\mathrm{d}, \mathrm{h}) \mathrm{Bi}$ 元素面分布图

Fig. 2 (a, e) Back-scattering electron (BSE) images and (b, f)Ge, (c, g)Te and (d, h) Bi elemental distributions of the polished surfaces for $(\mathrm{GeTe})_{n} \mathrm{Bi}_{2} \mathrm{Te}_{3}$ samples $((\mathrm{a}-\mathrm{d}) n=10$, (e-h) $n=14)$ 

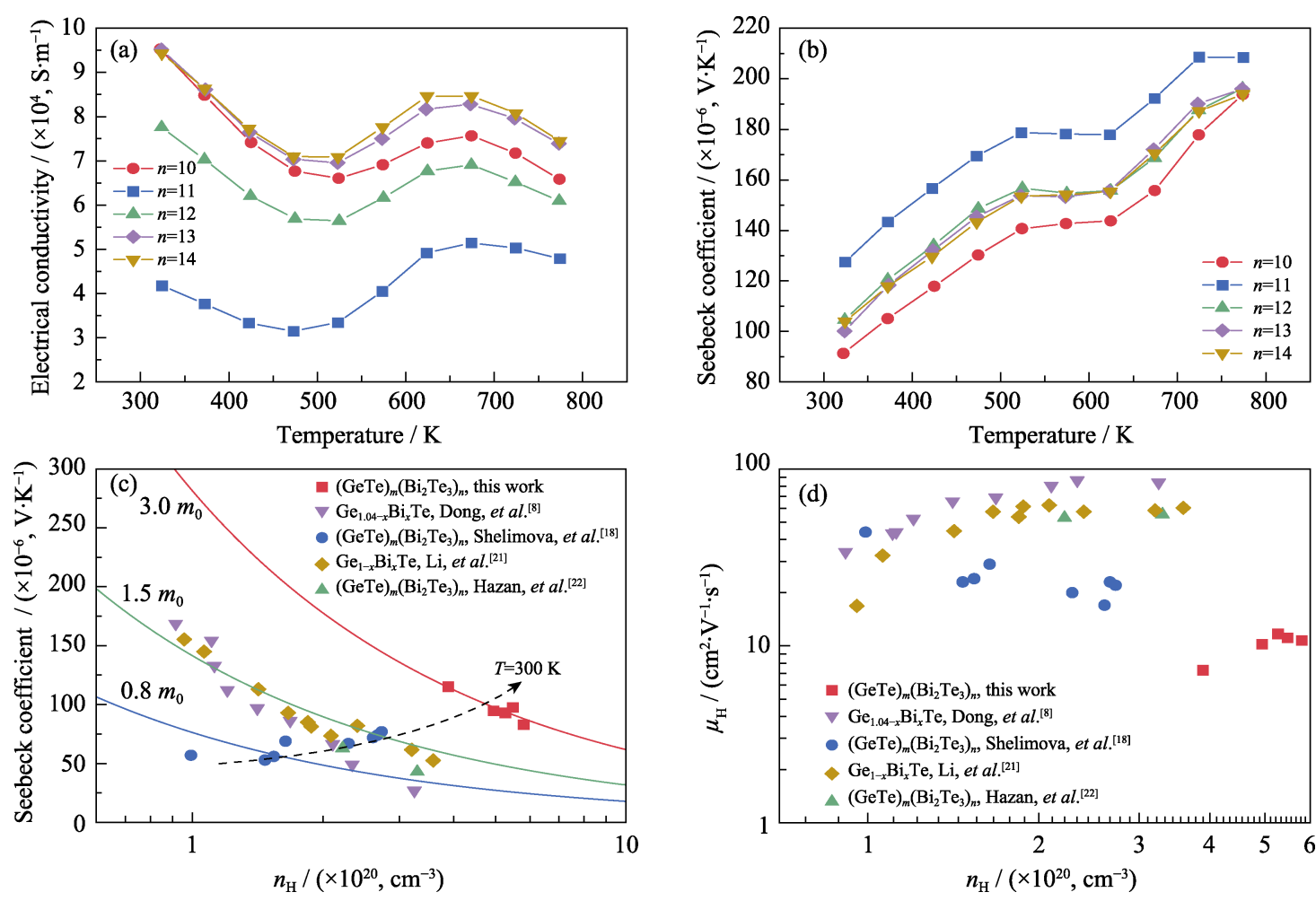

图 $3(\mathrm{GeTe})_{n} \mathrm{Bi}_{2} \mathrm{Te}_{3}$ 的 (a)电导率、(b)塞贝克系数随温度的变化曲线, (c)Pisarenko 曲线, (d)迁移率与载流子浓度的关系

Fig. 3 Temperature dependence of (a) electrical conductivity, (b) Seebeck coefficient, (c) Pisarenko plots,

(d) room temperature carrier mobility as a function of the carrier concentration for $(\mathrm{GeTe})_{n} \mathrm{Bi}_{2} \mathrm{Te}_{3}$

公式(1)中 $S$ 为塞贝克系数, $k_{\mathrm{B}}$ 为玻尔兹曼常数, $e$ 为 电子电量, $h$ 为普朗克常量, $m *$ 为有效质量, $n$ 为载流 子浓度。在图 3(c)中, 为了方便比较, 文献中报道的 单质 $\mathrm{Bi}$ 掺杂 $\mathrm{GeTe}$ 样品的数据也绘制在图中, 图中 实线为假定单抛物带结构和声学支声子散射占主导 的情况下，根据公式(1)在不同有效质量下计算得到 材料的塞贝克系数与载流子浓度的关系曲线, 可以 看出, 只掺杂 $\mathrm{Bi}$ 样品 ${ }^{[21]}$ 的有效质量随 $\mathrm{Bi}$ 掺杂量 $x$ 的变化不明显，载流子有效质量约为 $1.5 m_{0}$ 左右，而 对于 $(\mathrm{GeTe})_{n} \mathrm{Bi}_{2} \mathrm{Te}_{3}$ 系列样品, 本工作中 $n=10 \sim 14$ 系列 样品的有效质量在 $3.0 m_{0}$ 左右, 明显高于 Shelimova 等 ${ }^{[18]}$ 报道的 $n=2 \sim 9$ 系列样品, 有利于塞贝克系数的 提升，如图 3(c)中的黑色虚线所示。

需要指出的是本工作中 $\mathrm{Bi}$ 含量较低, 样品的载 流子浓度高于文献中的其他样品, 但由于较大的载 流子有效质量, 即使在较高载流子浓度的情况下样 品依然保持较高的塞贝克系数。图 3(d)为样品中载 流子迁移率与载流子浓度的关系, 可以看出采用单 质 $\mathrm{Bi}$ 掺杂样品的载流子迁移率明显高于以 $\mathrm{Bi}_{2} \mathrm{Te}_{3}$ 掺杂的样品, 这是由于 $(\mathrm{GeTe}){ }_{n} \mathrm{Bi}_{2} \mathrm{Te}_{3}(n=10 \sim 14)$ 的载 流子浓度较高, 载流子与载流子之间的散射加剧, 此外, 2 个 $\mathrm{Bi}$ 原子在 $\mathrm{Ge}$ 位发生置换，同时在晶格的 阳离子位置上产生一个空位，这些都会增强对载流 子的散射, 因此样品的迁移率较低, 在这些因素的
综合影响下, 最终 $(\mathrm{GeTe})_{n} \mathrm{Bi}_{2} \mathrm{Te}_{3}$ 系列样品呈现出较 低的电导率。

图 4 是 $(\mathrm{GeTe})_{n} \mathrm{Bi}_{2} \mathrm{Te}_{3}$ 的功率因子随温度的变化 曲线, 功率因子随着温度的升高显著增加, $n=13$ 的 样品在 $723 \mathrm{~K}$ 时获得最大的功率因子为 $2.88 \times$ $10^{-3} \mathrm{~W} \cdot \mathrm{m}^{-1} \cdot \mathrm{K}^{-2}$, 插图为室温下 $(\mathrm{GeTe})_{n} \mathrm{Bi}_{2} \mathrm{Te}_{3}$ 系列样 品的功率因子与载流子浓度的关系, 随着载流子浓 度的增加, 功率因子先上升后下降, 在载流子浓度 为 $5.5 \times 10^{20} \mathrm{~cm}^{-3}$ 时获得最佳功率因子, 达到 $1.02 \times$ $10^{-3} \mathrm{~W} \cdot \mathrm{m}^{-1} \cdot \mathrm{K}^{-2}$ 。

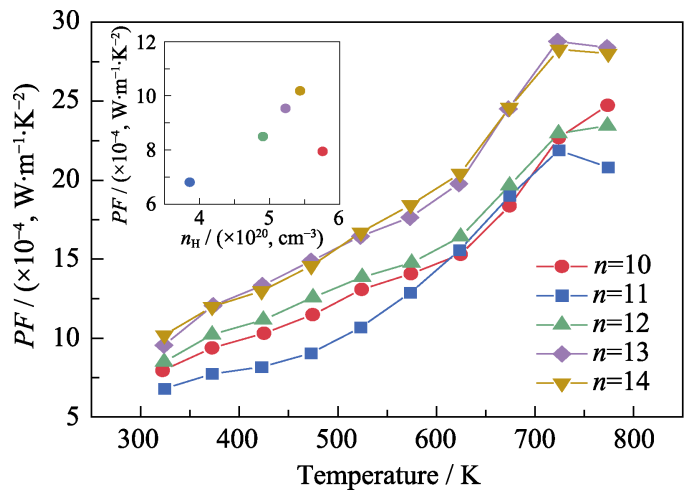

图 $4(\mathrm{GeTe})_{n} \mathrm{Bi}_{2} \mathrm{Te}_{3}$ 系列样品随温度变化的功率因子, 插图 为 $723 \mathrm{~K}$ 时系列样品的功率因子与载流子浓度的关系

Fig. 4 Temperature dependence of the power factor for $(\mathrm{GeTe})_{n} \mathrm{Bi}_{2} \mathrm{Te}_{3}$ compounds with inset showing the corresponding relationship between carrier concentration and power factor at $723 \mathrm{~K}$ 


\section{3 热输运性能及分析}

$(\mathrm{GeTe})_{n} \mathrm{Bi}_{2} \mathrm{Te}_{3}$ 系列样品的热导率和晶格热导率 随温度的变化曲线如图 5 所示。图 5(a)为 $(\mathrm{GeTe})_{n} \mathrm{Bi}_{2} \mathrm{Te}_{3}$ 系列样品的总热导率随温度的变化曲线, $(\mathrm{GeTe})_{n} \mathrm{Bi}_{2} \mathrm{Te}_{3}$ 系列样品的总热导率较本征的 $\mathrm{GeTe}$ 样品明显更低, 在室温下, $(\mathrm{GeTe})_{11} \mathrm{Bi}_{2} \mathrm{Te}_{3}$ 样品的总 热导率为 $1.06 \mathrm{~W} \cdot \mathrm{m}^{-1} \cdot \mathrm{K}^{-1}$, 而本征 $\mathrm{GeTe}$ 样品的热导 率为 $7.14 \mathrm{~W} \cdot \mathrm{m}^{-1} \cdot \mathrm{K}^{-1}$ 。此外, 与本征 $\mathrm{GeTe}$ 相比, $(\mathrm{GeTe})_{n} \mathrm{Bi}_{2} \mathrm{Te}_{3}$ 系列样品的总热导率随温度的升高缓 慢增大, 在 600 700 K 获得最大的热导率, 随着温 度的进一步升高, 热导率下降。在整个测试温度范围 内, $(\mathrm{GeTe})_{11} \mathrm{Bi}_{2} \mathrm{Te}_{3}$ 样品的热导率最低。材料的热导 率主要由晶格热导率和载流子热导率两部分组成:

$$
\kappa=\kappa_{\mathrm{e}}+\kappa_{\mathrm{L}}
$$

公式(2)中, $\kappa_{\mathrm{L}}$ 为样品的晶格热导率, $\kappa_{\mathrm{e}}$ 为载流子 热导率, 材料的载流子热导率可以根据 WiedemannFranz 定律计算得到，表示为:

$$
\kappa_{\mathrm{e}}=L \sigma T
$$

公式(3)中 $\sigma$ 为电导率, $L$ 为洛伦兹常数, 假设为单 抛物线形能带结构下, 洛伦兹常数可以表示为:

$L=\left(\frac{k_{\mathrm{B}}}{e}\right)^{2}\left(\frac{\left(r+\frac{7}{2}\right) F_{r+5 / 2}(\eta)}{\left(r+\frac{3}{2}\right) F_{r+1 / 2}(\eta)}-\left[\frac{\left(r+\frac{5}{2}\right) F_{r+3 / 2}(\eta)}{\left(r+\frac{3}{2}\right) F_{r+1 / 2}(\eta)}\right]^{2}\right)$

公式(4)中 $\eta=E_{F} /\left(k_{\mathrm{B}} T\right)$ 为简约费米能级, $r$ 为散射因 子。当载流子散射机制主要为声学声子散射时, $r$ 取 $-1 / 2$ 。 $\eta$ 可以根据实测的塞贝克系数从公式(5)导出:

$$
S= \pm \frac{k_{\mathrm{B}}}{e}\left(\frac{\left(r+\frac{5}{2}\right) F_{r+3 / 2}(\eta)}{\left(r+\frac{3}{2}\right) F_{r+1 / 2}(\eta)}-\eta\right)
$$

公式(4 5)中的 $F_{n}(\eta)$ 为费米积分, 可以表示为:

$$
F_{n}(\eta)=\int_{0}^{\infty} \frac{x^{n}}{1+e^{x-\eta}} \mathrm{d} x
$$

根据实测的塞贝克系数数据, 结合公式(4 6), 可以计算出样品随温度变化的洛伦兹常数, 最终根 据公式(2,3)得到晶格热导率。图 5(b)为 $(\mathrm{GeTe})_{n} \mathrm{Bi}_{2} \mathrm{Te}_{3}$ 系列样品的晶格热导率随温度的变化曲线。在 $525 \mathrm{~K}$ 以下, 系列样品的晶格热导率几乎保持不变, 随着温度的升高, 晶格热导率迅速下降, 在 $723 \mathrm{~K}$ 左右获得最小值, $(\mathrm{GeTe})_{13} \mathrm{Bi}_{2} \mathrm{Te}_{3}$ 样品在整个温度范 围内的晶格热导率最低, 室温下其晶格热导率为 $0.84 \mathrm{~W} \cdot \mathrm{m}^{-1} \cdot \mathrm{K}^{-1}$ 。在 $723 \mathrm{~K}$ 时, 其晶格热导率下降到 $0.51 \mathrm{~W} \cdot \mathrm{m}^{-1} \cdot \mathrm{K}^{-1}$ 。
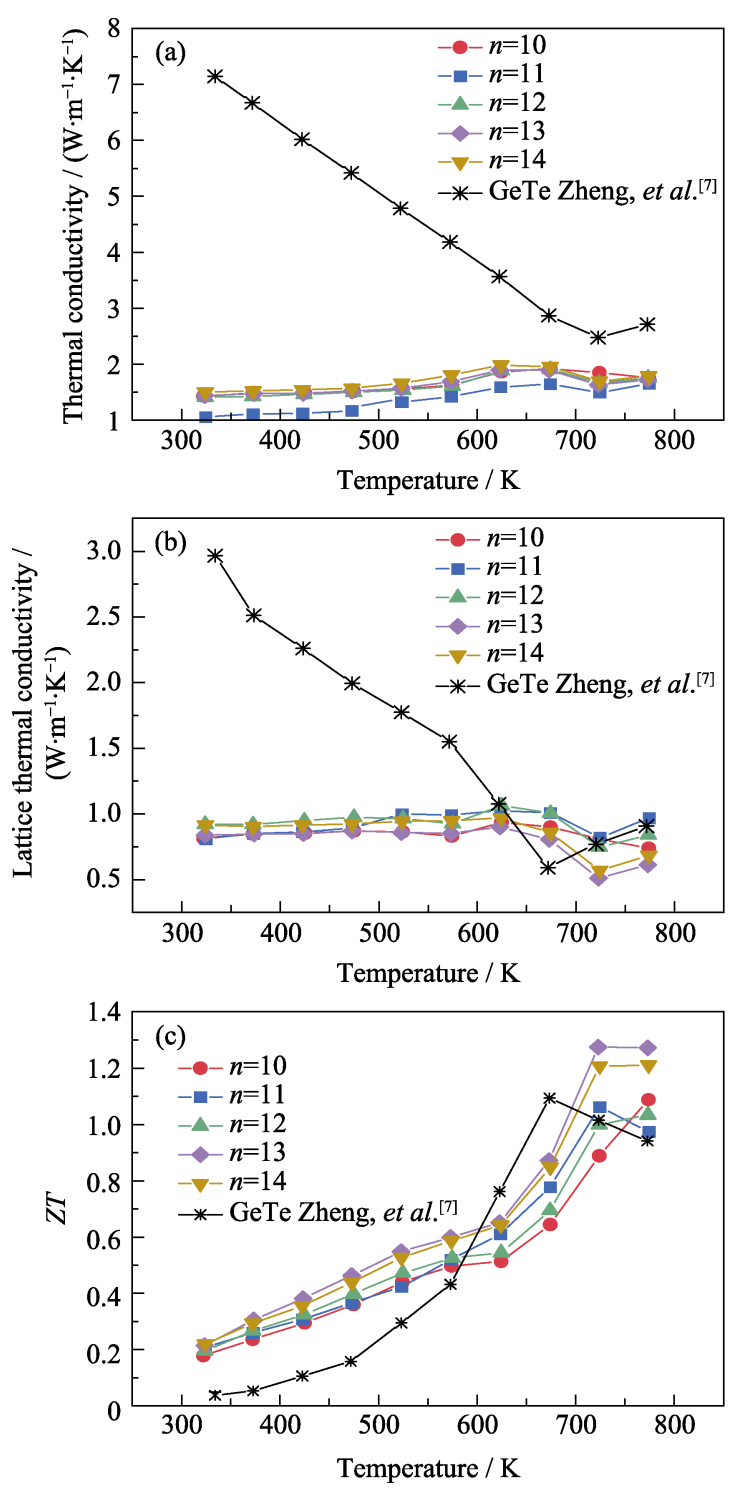

图 $5(\mathrm{GeTe})_{n} \mathrm{Bi}_{2} \mathrm{Te}_{3}$ 系列样品随温度变化的(a)总热导率和 (b) 晶格热导率, (c)样品的热电性能优值 $Z T$ 随温度变化曲线 Fig. 5 Temperature dependence of (a) total thermal conductivity $\kappa$, (b) lattice thermal conductivity $\kappa_{\mathrm{L}}$ and (c) figure of merit $Z T$ for $(\mathrm{GeTe})_{n} \mathrm{Bi}_{2} \mathrm{Te}_{3}$

利用实测的电导率、塞贝克系数和热导率, 计 算材料的热电性能优值 $Z T$, 图 5(c)为样品的热电性 能优值随温度的变化曲线, 随着温度的升高, $Z T$ 值 逐渐增大, $n=13$ 的样品在 $723 \mathrm{~K}$ 下获得最大的 $Z T$ 值 1.27 , 与本征 $\mathrm{GeTe}$ 样品 $673 \mathrm{~K}$ 下的最大 $Z T$ 值 $1.09^{[7]}$ 相比提高了 $16 \%$, 热电性能的提升主要来源于掺杂 $\mathrm{Bi}_{2} \mathrm{Te}_{3}$ 后, 材料的热导率大幅度降低。

\section{3 结论}

本研究采用熔融、退火结合放电等离子活化烧 结(SPS)工艺成功制备了 $(\mathrm{GeTe})_{n} \mathrm{Bi}_{2} \mathrm{Te}_{3}$ 系列单相样品, 采用 XRD 和 EDS 分析结果表明所获得的样品具有 
菱方相结构且组成分布均匀。通过调控 $\mathrm{Bi}_{2} \mathrm{Te}_{3}$ 和 GeTe 相对含量显著提高了载流子有效质量, $(\mathrm{GeTe}){ }_{11} \mathrm{Bi}_{2} \mathrm{Te}_{3}$ 样品在 $723 \mathrm{~K}$ 下获得的最大塞贝克系数 为 $208 \mu \mathrm{V} \cdot \mathrm{K}^{-1},(\mathrm{GeTe}){ }_{13} \mathrm{Bi}_{2} \mathrm{Te}_{3}$ 样品在约 $723 \mathrm{~K}$ 下获得 最大的功率因子为 $2.88 \times 10^{-3} \mathrm{~W} \cdot \mathrm{m}^{-1} \cdot \mathrm{K}^{-2}$ 。同时, 掺 杂 $\mathrm{Bi}_{2} \mathrm{Te}_{3}$ 显著增强了缺陷对声子的散射, 材料的晶 格热导率显著降低, $(\mathrm{GeTe})_{13} \mathrm{Bi}_{2} \mathrm{Te}_{3}$ 样品在整个温度 范围内具有最低的晶格热导率, 在约 $723 \mathrm{~K}$ 时, 其 晶格热导率为 $0.51 \mathrm{~W} \cdot \mathrm{m}^{-1} \cdot \mathrm{K}^{-1}$ 。由于样品较低的热导 率和较大的功率因子, 在 $723 \mathrm{~K}$ 下该样品获得最大 $Z T$ 值 1.27 , 较本征 GeTe 样品提高了 $16 \%$ 。

\section{参考文献:}

[1] ROWE D M. CRC Handbook of thermoelectrics. New York: CRC Press, 1995: 7-17.

[2] ROYCHOWDHURY S, SAMANTA M, PERUMAL S, et al. Germanium chalcogenide thermoelectrics: electronic structure modulation and low lattice thermal conductivity. Chemistry of Materials, 2018, 30(17): 5799-5813.

[3] HONG M, ZOU J, CHEN Z G. Thermoelectric GeTe with diverse degrees of freedom having secured superhigh performance. Advanced Materials, 2019, 31(14): 1-23.

[4] PERUMAL S, ROYCHOWDHuRY S, BISWAS K. High performance thermoelectric materials and devices based on GeTe. Journal of Materials Chemistry C, 2016, 4(32): 7520-7536.

[5] OKAMOTO H. Ge-Te (Germanium-Tellurium). Journal of Phase Equilibria, 2000, 21(5): 496.

[6] LEWIS J E. The defect structure of non-stoichiometric germanium telluride from magnetic susceptibility measurements. Physica Status Solidi (B), 1970, 38(1): 131-140.

[7] ZHENG Z, SU X, DENG R, et al. Rhombohedral to cubic conversion of GeTe via MnTe alloying leads to ultralow thermal conductivity, electronic band convergence, and high thermoelectric performance. Journal of the American Chemical Society, 2018, 140(7): 2673-2686.

[8] DONG J, SUN F H, TANG H, et al. Medium-temperature thermoelectric GeTe: vacancy suppression and band structure engineering leading to high performance. Energy and Environmental Science, 2019, 12(4): 1396-1403.

[9] PERUMAL S, ROYCHOWDHURY S, BISWAS K. Reduction of thermal conductivity through nanostructuring enhances the thermoelectric figure of merit in $\mathrm{Ge}_{1-x} \mathrm{Bi}_{x} \mathrm{Te}$. Inorganic Chemistry Frontiers, 2016,
3(1): 125-132.

[10] PERUMAL S, BELLARE P, SHENOY U S, et al. Low thermal conductivity and high thermoelectric performance in $\mathrm{Sb}$ and $\mathrm{Bi}$ codoped GeTe: complementary effect of band convergence and nanostructuring. Chemistry of Materials, 2017, 29(24): 10426-10435.

[11] PERUMAL S, ROYCHOWDHURY S, NEGI D S, et al. High thermoelectric performance and enhanced mechanical stability of P-type $\mathrm{Ge}_{1-x} \mathrm{Sb}_{x} \mathrm{Te}$. Chemistry of Materials, 2015, 27(20): 7171-7178.

[12] WUTTIG M, YAMADA N. Phase-change materials for rewriteable data storage. Nature Materials, 2007, 6(11): 824-832.

[13] ROSENTHAL T, SCHNEIDER M N, STIEWE C, et al. Real structure and thermoelectric properties of GeTe-rich germanium antimony tellurides. Chemistry of Materials, 2011, 23(19): 4349-4356.

[14] XU X, XIE L, LOU Q, et al. Boosting the thermoelectric performance of pseudo-layered $\mathrm{Sb}_{2} \mathrm{Te}_{3}(\mathrm{GeTe})_{n}$ via vacancy engineering. Advanced Science, 2018, 5(12): 1801514-1801520.

[15] KOSUGA A, NAKAI K, MATSUZAWA M, et al. Crystal structure, microstructure, and thermoelectric properties of $\mathrm{GeSb}_{6} \mathrm{Te}_{10}$ prepared by spark plasma sintering. Journal of Alloys and Compounds, 2015, 618: $463-468$.

[16] ROSENTHAL T, URBAN P, NIMMRICH K, et al. Enhancing the thermoelectric properties of germanium antimony tellurides by substitution with selenium in compounds $\mathrm{Ge}_{n} \mathrm{Sb}_{2}\left(\mathrm{Te}_{1-x} \mathrm{Se}_{x}\right)_{n+3}(0 \leqslant$ $x \leqslant 0.5 ; n \geqslant 7)$. Chemistry of Materials, 2014, 26(8): 2567-2578.

[17] SCHNEIDER M N, BIQUARD X, STIEWE C, et al. From metastable to stable modifications - in situ laue diffraction investigation of diffusion processes during the phase transitions of $(\mathrm{GeTe})_{n} \mathrm{Sb}_{2} \mathrm{Te}_{3}(6<n<15)$ crystals. Chemical Communications, 2012, 48(16): 2192-2194.

[18] SHELIMOVA L E, KONSTANTINOV P P, KARPINSKY O G, et al. X-ray diffraction study and electrical and thermal transport properties of the $n \mathrm{GeTe} \cdot m \mathrm{Bi}_{2} \mathrm{Te}_{3}$ homologous series compounds. Journal of Alloys and Compounds, 2001, 329(1/2): 50-62.

[19] LI J, ZHANG C, FENG Y, et al. Effects on phase transition and thermoelectric properties in the $\mathrm{Pb}$-doped $\mathrm{GeTe}-\mathrm{Bi}_{2} \mathrm{Te}_{3}$ alloys with thermal annealing. Journal of Alloys and Compounds, 2019, 808: 151747-151755.

[20] WU D, ZHAO L D, HAO S, et al. Origin of the high performance in $\mathrm{GeTe}$-based thermoelectric materials upon $\mathrm{Bi}_{2} \mathrm{Te}_{3}$ doping. Journal of the American Chemical Society, 2014, 136(32): 11412-11419.

[21] LI J, CHEN Z, ZHANG X, et al. Electronic origin of the high thermoelectric performance of GeTe among the P-type group IV monotellurides. NPG Asia Materials, 2017, 9(3): e353-1-8.

[22] HAZAN E, MADAR N, PARAG M, et al. Effective electronic mechanisms for optimizing the thermoelectric properties of GeTe-rich alloys. Advanced Electronic Materials, 2015, 1(11): 1-7. 